OPEN ACCESS

Edited by:

Stefania Tacconelli,

University of Studies G. d'Annunzio

Chieti and Pescara, Italy

Reviewed by:

Gurudutt Pendyala,

University of Nebraska Medical Center,

United States

Soon Yew Tang,

University of Pennsylvania,

United States

*Correspondence:

Chunmeng Shi

shicm@tmmu.edu.cn

Lin Xie

xielin@hospital.cqmu.edu.cn

Specialty section:

This article was submitted to Inflammation Pharmacology,

a section of the journal

Frontiers in Pharmacology

Received: 07 April 2020

Accepted: 25 June 2020

Published: 31 July 2020

Citation:

Li X, Leng $Y$, Jiang $Q$, Wang Z, Luo $P$,

Zhang $C$, Chen L, Wang $Y$, Wang $H$,

Yue X, Shen C, Zhou Y, Shi C and Xie L

(2020) Eye Drops of Metformin

Prevents Fibrosis After Glaucoma

Filtration Surgery in Rats via Activating

AMPK/Nrf2 Signaling Pathway.

Front. Pharmacol. 11:1038.

doi: 10.3389/fphar.2020.01038

\section{Eye Drops of Metformin Prevents Fibrosis After Glaucoma Filtration Surgery in Rats via Activating AMPK/ Nrf2 Signaling Pathway}

\author{
Xueru Li ${ }^{1}$, Yu Leng ${ }^{1}$, Qingzhi Jiang ${ }^{2}$, Ziwen Wang ${ }^{3}$, Peng Luo ${ }^{3}$, Chi Zhang ${ }^{3}$, Long Chen ${ }^{3}$, \\ Yawei Wang ${ }^{3}$, Huilan Wang ${ }^{2}$, Xiaofeng Yue ${ }^{4}$, Chongxing Shen ${ }^{4}$, Yuanlinhan Zhou ${ }^{5}$, \\ Chunmeng Shi ${ }^{3 *}$ and Lin Xie ${ }^{1 *}$ \\ ${ }^{1}$ Department of Ophthalmology, The Third Affiliated Hospital of Chongqing Medical University (Gener Hospital), Chongqing, \\ China, ${ }^{2}$ Department of Oncology, The Affiliated Hospital of Southwest Medical University, Luzhou, China, ${ }^{3}$ Institute of \\ Rocket Force Medicine, State Key Laboratory of Trauma, Burns and Combined Injury, Third Military Medical University, \\ Chongqing, China, ${ }^{4}$ Department of Urology, The Third Affiliated Hospital of Chongqing Medical University (Gener Hospital), \\ Chongqing, China, ${ }^{5}$ Chongqing Industry Polytechnic College, Chongqing, China
}

Metformin has effective therapeutic effects in anti-tumor and anti-fibrotic diseases. However, how the antifibrotic effect of metformin in the eye and how it is transferred are still unclear. Here, the eye drop of metformin treatment was studied in Sprague-Dawley (SD) rats of glaucoma filtrating surgery (GFS). Rats were administered randomly bilateral drops: control group (without surgery), GFS group, metformin group or mitomycin C (MMC) group (sponge application intraoperatively, 0.02\%). Bleb features and intraocular pressure (IOP) were assessed for postoperative week 4. Metformin effectively inhibited fibrosis and improved the surgical outcomes of GFS. In vitro, we found that the degree of oxidative stress and fibrosis in metformin pretreated-Human Conjunctival Fibroblasts (HConFs) were reduced; the pro-fibrotic response of HConFs were decreased by inducing macrophagic polarity changes. Besides, the inhibition of nuclear factor erythroid 2-related factor 2 (Nrf2)/ AMP-activated protein kinase (AMPK) and the competition of organic cation transporters (OCTs) effectively reduced the anti-fibrotic capability of metformin. Together, this experiment indicates that metformin enters into HConFs cell with OCTs, which can protect against filtrating blebs scar formation in SD rats of GFS via activating AMPK/Nrf2 axis and the downregulation of profibrogenic and inflammatory biomarkers.

Keywords: glaucoma filtration surgery (GFS), metformin, fibrosis, AMPK/Nrf2, oxidative stress, macrophages, organic cation transporters (OCTs)

\section{INTRODUCTION}

Glaucoma filtration surgery (GFS) is the most efficacious option for uncontrolled-IOP glaucoma besides medicine and laser treatment (Lanzl et al., 2016). However, with the destruction of the tissue vasculature, the stimulation of exudate and hormones on conjunctival fibroblasts, these are risk factors for pathological filtration of fibrotic fibrosis. Postoperative scar formation is the major 
obstacle to increased success (Zada et al., 2018; Addicks et al., 1983; Schlunck et al., 2016). Currently, anti-inflammatory medication and antimetabolites such as Mitomycin C (MMC), 5-fluorouracil (5-FU) and steroids are widely applied in clinical, but complications (corneal toxicity, hypotony, wound leak and even infectious endophthalmitis) limit their applications (Cabourne et al., 2015). Therefore, further improvements and strategies are urgently needed to better control filtrating blebs fibrosis.

Recently, accumulated literatures reported that the therapeutic effects of metformin in anti-tumor and anti-fibrotic diseases such as breast cancer (Patterson et al., 2018; Sharma and Kumar, 2018), infertility (Sivalingam et al., 2014) and KRAS/ LKB1 co-mutated NSCLC (Moro et al., 2018) and fibrosis (Biondo et al., 2018; Juban et al., 2018). Metformin exhibited pleiotropic mechanisms for cell protection including mitochondrial integrity, proliferation, inflammatory response, oxidative stress and ER stress (Sato et al., 2016; Sharma and Kumar, 2017). Moreover, metformin is an AMPK activator as a crucial sensor of cellular bioenergy, which can control the switch from anabolic to catabolic. It was resistant to stress and maintained the balance of cellular metabolism when sensing and decomposing mitochondrial ROS. (Hardie et al., 2012; Quarta et al., 2019), which were risk factors for pathologic fibrotic processes (Salminen and Kaarniranta, 2012). Not only that effects of oral administration of metformin on ocular disease have also attracted widespread attention, the preclinical studies showed that metformin exerted protective effects on reducing risks of developing open angle glaucoma (OAG) (Lin et al., 2015) and had obvious anti-inflammatory and antiangiogenic effects on retinal vascular hyperplasia (Han et al., 2018b). However, it is not clear how the antifibrotic effect of metformin in the eye and how it is transferred.

In this study, the experimental results showed that eye drops of metformin could effectively reduce fibrosis and prolonged bleb survival in SD rats GFS models postoperative 4 weeks. Mechanistically, metformin entered into Human Conjunctival Fibroblasts (HConFs) by organic cation transporters (OCTs), which reduced HConFs fibrosis via a mechanism involving oxidative stress promoting and anti-fibrosis in TGF- $\beta 2$ induced collagen production. Our results highlight the potential of using metformin eye drops to treat patients after GFS.

\section{METHODS AND MATERIALS}

\section{Reagents and Antibodies}

The TGF- $\beta 2$ cytokine was purchased from Pepro Tech. Glyceraldehyde-3-phosphatedehydrogenase (GAPDH), horseradish peroxidase (HRP)-conjugated secondary antibodies, RIPA buffer, H2DCF-DA, BCA kit, western blocking buffer, $\mathrm{H}_{2}$ DCF-DA were the products of Beyotime (China). The nucleoside 5-aminoimidazole-4carboxamide riboside (AICAR), metformin hydrochloride, Phorbol 12-myristate 13-acetate (PMA), ML385 (an Nrf2 inhibitor) (CAS No.: 846557-71-9), Atropine (CAS No.: 51-55-8) and Quinidine
(CAS No.: 56-54-2) were acquired from MedChemExpress (MCE) (China). Antibodies against AKT, p-AKT, AMPK and p-AMPK (172) were purchased from CST. Antibodies against superoxide dismutase 1 (SOD1), SOD2, $\gamma$-glutamyl cysteine synthetase $(\gamma$-GCS), Catalase (CAT), smad $2 / 3$ and $\beta$-actin were purchased from Proteintech. Antibodies against Nrf2, $\alpha$-smooth muscle actin $(\alpha$ SMA) (ab5694), Osteopontin (OPN) (ab8448), collagen-I, p-smad2/ 3 and fibronectin (ab34710) were purchased from Abcam. Antibody against p-Nrf2(S40) was provided by Santa Cruz Biotechnology. The cell culture reagents were the products of Gibco Laboratories (CAS No.:16000-044).

\section{Cell Culture}

Primary HConFs (or THP1) cell lines were acquired form ATCC (USA) for this study. The HConFs (or THP1) was cultured in DMEM (or RIPM 1640) containing 10\% FBS (Gibco, USA), 1\% cyan-streptomycin at $37^{\circ} \mathrm{C}$ with $5 \% \mathrm{CO}_{2}$. The serum-starved $(12$ h) HConFs was used for cell experiments.

\section{Rat Glaucoma Filtration Surgery Models and Treatments}

Thirty-two female SD rats (6-8 weeks, 200-250 g) were used. The animals were anaesthetized by intraperitoneal injection of overdose $10 \%$ Chloral Hydrate $(1 \mathrm{ml} / 100 \mathrm{~g})$. Topical anesthesia ( $0.5 \%$ oxybutyn hydrochloride) was used before the operation. GFS was performed as previously described (Sherwood et al., 2004). A conjunctival flap based on the limbus was formed 3-5 $\mathrm{mm}$ behind the limbus. Then, inserted a $25 \mathrm{G}$ needle into anterior chamber (AC) to create a full-thickness scleral tunnel. Afterwards, a beveled 30-G microcannula was inserted into the scleral tunnel. The microcannula was fixed to the limbus. Finally, 10-0 (0.1 metric) Ethicon monofilament nylon sutures were used to close the conjunctiva. Random bilateral drug administration to rats: control group (without surgery), GFS group (saline, with surgery), metformin group $(7.85 \mathrm{mM} / \mathrm{drop}$, four times/day and postoperative 2 weeks) $(\mathrm{N}=5)$ and MMC group (sponge application, $0.02 \%$ ), which were evaluated at a pre-set endpoints.

\section{Histological Examinations}

On postoperative 14 days, twenty female SD rats were euthanized. Removing their surgical eyeball, A small opening was cut away from surgical wounds, then soaked the eyeball in $4 \%$ formaldehyde solution (48 h). The tissues were dehydrated, encapsulated, sliced, and then used for $\mathrm{H} \& \mathrm{E}$ and Masson trichrome staining. Tissue sections were also used for immunofluorescence staining. The sections were dewaxed, rehydrated, and treated with $3 \%$ hydrogen peroxide at room temperature for $10 \mathrm{~min}$. Then, the sections were treated with $0.4 \%$ pepsin at $37^{\circ} \mathrm{C}$ for $30 \mathrm{~min}$ to recover the antigen, followed by blocking with $1 \%$ goat serum albumin at room temperature for 40 $\min$ (Li et al., 2020). After incubating with primary antibodies $(\alpha-$ SMA, vimentin and type 1 collagen, 1:200) at $4^{\circ} \mathrm{C}$ overnight, the sections were incubated with an appropriate biotinylated secondary antibody at $37^{\circ} \mathrm{C}$ for $1 \mathrm{~h}$, and the image was captured under an optical microscope (Olympus Inc., Tokyo, Japan). 


\section{Transwell Co-Culture Assay}

The bottom membrane of the Trans-well chamber was coated with serum $\left(37^{\circ} \mathrm{C}, 1 \mathrm{~h}\right)$, followed by washing with PBS. Collected THP1 cells in logarithmic phase, adjusted the cell density to $1 \times$ $10^{5}$ cells $/ \mathrm{ml}$ with $10 \%$ FBS medium, added $500 \mu \mathrm{l}$ of cell suspension to the lower chamber of the 24-well plate, and induced with PMA (100 nM) for two days. On third day, macrophages M1, M2 different inducers LPS, IL-4 + IL-13 were added and cultured for $24 \mathrm{~h}$ under the intervention of metformin $(2 \mathrm{mM})$. The HConFs $\left(1 \times 10^{5}\right.$ cells $)$ was cultured (serum-free medium) in a 24 -well plate compartment and cocultured for different time periods. Removed the trans-well chamber, discard the culture medium in the wells, gently wiped the upper non-migrating cells with a cotton swab, fixed with paraformaldehyde fixative $(20 \mathrm{~min})$ followed by washing with water carefully after staining with crystal violet for $20 \mathrm{~min}$. Cells were randomly observed in five fields under a $400 \times$ microscope and counted.

\section{Western Blotting Analysis}

Total protein was extracted and quantified by BCA kit. Transferred protein of different molecular weight to polyvinylidene fluoride membrane by SDS-PAGE (Millipore) followed by blocking with blocking buffer $\left(37^{\circ} \mathrm{C}, 1 \mathrm{~h}\right)$. The membranes were incubated with a primary antibody $(\gamma-\mathrm{GCS}, \mathrm{SOD} 1 / 2, \mathrm{CAT}, \mathrm{p}-\mathrm{Nrf2}$ (S40), Nrf2, $\operatorname{smad} 2 / 3, \mathrm{p}$-smad2/3, vimentin, $\alpha$-SMA, fibronectin, Akt/p-Akt, AMPK/-AMPK, collagen 1 (ECM deposition level) and GAPDH, 1:1,000) overnight at $4{ }^{\circ} \mathrm{C}$. At room temperature, the blots and HRPconjugated secondary antibody (CST, 1:1,000) were washed three times (15 min/times) with TBST at the beginning and end of the 1hour incubation on a shaker. An enhanced chemiluminescence detection system (Bio-Rad Laboratories) and Image J software (Bethesda, Maryland, USA) were used, the intensity of bands normalized to GADPH statistics was observed and analyzed (Li et al., 2020).

\section{Real-Time PCR}

We isolated total mRNA from $\mathrm{HConFs}$ cell with TRIzol ${ }^{\circledR}$ reagent. A Prime Script TMRT kit with cDNA Eraser (\#K1622) was used for reverse transcription. qRT-PCR was performed by SYBR Green qPCR master mix (Takara, Japan). The total reaction volume was $20 \mu \mathrm{l}=10 \mu \mathrm{l}$ (SYBR solution, 2.5× Real Master Mix) $+0.5 \mu \mathrm{l}$ (Forward primer, $10 \mu \mathrm{mol} / \mathrm{L})+0.5 \mu \mathrm{l}$ (Reverse primer, $10 \mu \mathrm{mol} / \mathrm{L}$ ) $+1 \mu \mathrm{l} \mathrm{cDNA}+8 \mu \mathrm{l}$ (ultra-pure water) (Li et al., 2020). Reaction conditions: Total mRNA was pre-denatured at $95^{\circ} \mathrm{C}$ for $10 \mathrm{~min}$, denatured at $95^{\circ} \mathrm{C}$ for $15 \mathrm{~s}$, annealed at $62{ }^{\circ} \mathrm{C}$ for $30 \mathrm{~s}$, extended at $68{ }^{\circ} \mathrm{C}$ for $30 \mathrm{~s}$, and 50 cycles. The $2^{-\Delta \Delta \mathrm{Ct}}$ method was used to calculating gene levels and normalize relative to GAPDH (Li et al., 2020).

\section{Cell Viability Assay}

The cells suspension was inoculated into 96 -well plates $\left(4 \times 10^{3}\right.$ cells/well) and preconditioned by TGF- $\beta 2$ with appear or without appear metformin (2 mM) treatment for 24, 48 and $72 \mathrm{~h}$. Then, HConFs were cultured in $100 \mu \mathrm{l}$ fresh complete medium (48 h) and then added $10 \mu \mathrm{l} \mathrm{CCK-8} \mathrm{reagent/well.} \mathrm{After} \mathrm{incubating} \mathrm{for} 1 \mathrm{~h}$ at $37^{\circ} \mathrm{C}$, the absorbance values were measured with a microplate reader (Multiskan Go Multimode Reader, Thermo Scientific).

\section{Measurement of Reactive Oxygen Species}

According to the previous experimental method (Li et al., 2020), to assess the oxidative stress level of HConFs, cells were planted into 6-well plates $\left(4 \times 10^{4}\right.$ cells $\left.^{-1}\right)$. After different treatment, the wells were decanted and washed two times. A $500 \mu \mathrm{l}$ reaction solution H2DCF-DA $\left(5 \times 10^{-6} \mathrm{~m}, 1: 1,000\right) /$ MitoSOX Red $(5 \mu \mathrm{M}$, CAS No.:M36008) for mitochondrial ROS evaluation was incubated at $37{ }^{\circ} \mathrm{C}$ for $20 \mathrm{~min}$ in the dark. Next, trypsindigested-HConFs were resuspended in $150 \mu \mathrm{l}$ with PBS. Then the signaling was tested by polychromatic flow cytometry and the data were analyzed (Flow-Jo_V10 software).

\section{Confocal Microscopy Imaging}

HConFs cells were seeded on $14 \mathrm{~mm}$ glass coverslips of 24-well plates and incubated for $24 \mathrm{~h}$ after different treatments. Slides were washed (two times) and fixed in $4 \%$ paraformaldehyde solution at $4^{\circ} \mathrm{C}$ overnight. It was blocked with $1 \%$ goat serum $\left(1 \mathrm{~h}, 37^{\circ} \mathrm{C}\right)$ after infiltration with $0.1 \%$ Triton-X100 (Li et al., 2020). Primary antibody fibronectin (1:200) was incubated at $4^{\circ}$ C overnight. Next, the slides were washed with PBS and incubated with AlexaFluor ${ }^{\circledR}$ 594/AlexaFluor ${ }^{\circledR} 488$-conjugated secondary antibody (1:500) for $40 \mathrm{~min}$ at $37^{\circ} \mathrm{C}$ in the dark followed by DAPI staining (2 $\mathrm{min})$. A fluorescence microscope (laser scanning confocal microscope, Leica) was used to capture the images and analyze them.

\section{Co-Culture Analysis}

To analyze the role of macrophages of different polarities on fibrosis of HConFs cells under the intervention of metformin $(2 \mathrm{mM})$. Collected THP1 cells in logarithmic phase, adjust the cell density to $1 \times 10^{5}$ cells $/ \mathrm{ml}$ with $10 \%$ FBS medium, added $2 \mathrm{~mL}$ of cell suspension to the higher chamber of the 6-well plates, and induced with PMA (100 nM) for two days. On third day, M0 macrophage was induced by LPS or IL-4/IL-13 (100 ng/ml or $20 \mathrm{ng} /$ $\mathrm{ml}$ ) into macrophages M1, M2 and cultured for $24 \mathrm{~h}$ under the intervention of metformin $(2 \mathrm{mM})$, and then the HConFs $\left(1 \times 10^{5}\right.$ cells) were treated with serum-free medium in a 6-well plates compartment and were co-cultured after different time periods. Collected the HConFs in the lower chamber of the 6-well plates, and performed $\mathrm{qRT}-\mathrm{PCR} /$ western blotting experiments to detect the fibrosis and proliferation levels of HConFs cells.

\section{Statistical Analysis}

All results were presented as mean \pm standard deviation. Oneway analysis of variance was applied to determine significance among groups. Statistical significance was set at ${ }^{\star} / \# \mathrm{p}<0.05$, ${ }^{* *} / \# \# \mathrm{p}<0.05$ and ${ }^{* *} / \# \# \# \mathrm{p}<0.01$. All the statistical analyses were performed using SPSS13.0 software (SPSS, USA). 


\section{RESULTS}

\section{Metformin Efficiently Prolongs Filtering Bleb Survival in Rats of GFS}

Accumulated studies reported that metformin has therapeutic effects on non-diabetic diseases. However, the antifibrotic role of metformin in ocular diseases is not clear. In this study, the role of topical metformin treatment was studied in SD rats of GFS (Figure 1A). The slit lamp inspection was to assess typical appearances of bubbles in SD rats' model at the 3, 7, 14 and 28 days postoperative endpoints (Supplemental Figure S1A). Representative images showed that metformin group (7.85 $\mathrm{mM} / \mathrm{drops}$ (an average of $30 \mathrm{ul} / \mathrm{drop}$ ), four times/day) significantly prolonged bleb survival. But the bubbles were flat, small and vascularized in GFS group. There were lager areas of functional filtration blebs in metformin group (Figure 1B). There is a higher intraocular pressure (IOP) in metformin group than MMC group and GFS group on 28 day postoperatively (Supplemental Figure S1B). Moreover, H\&E/ Masson's trichrome staining were used to assess tissue sections. Representative images revealed that the collagen deposition in metformin group was significantly decreased than GFS group (Figures 1C, D) (as black arrows showed). Furthermore, Masson's trichrome staining of surgical eyes in GFS group almost showed server scars at the surgical site, including more evidences of collagen deposition in surgical sites than MMC/
A

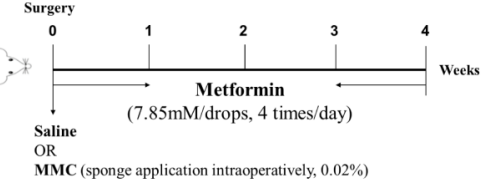

B

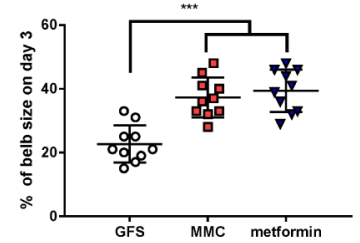

D
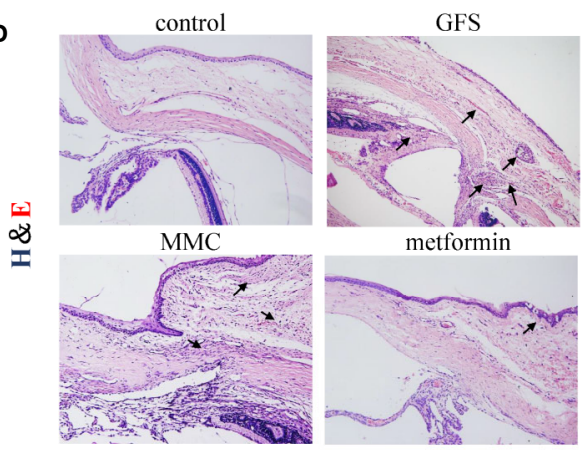

F

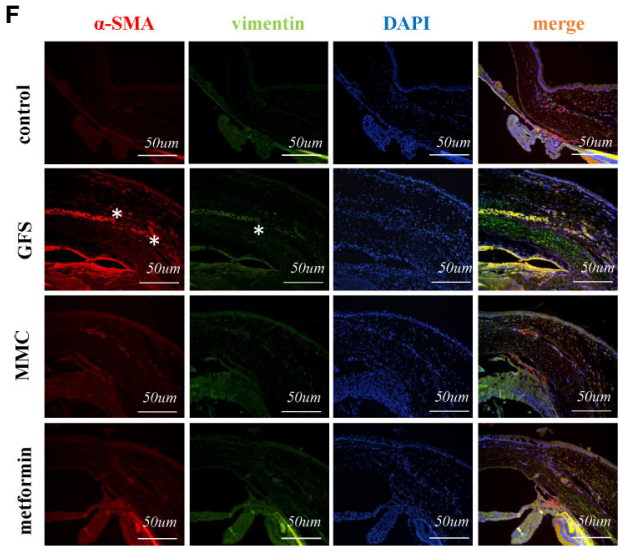

C

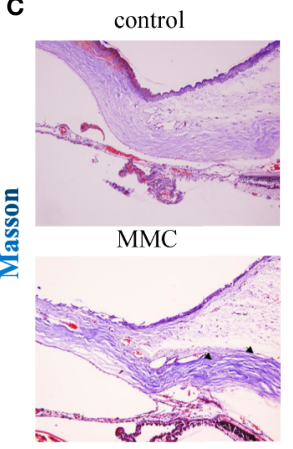

GFS

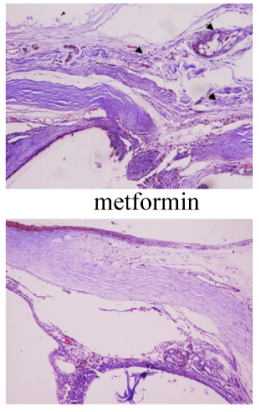

E

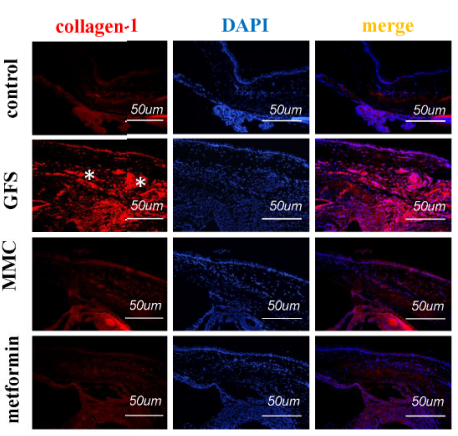

G

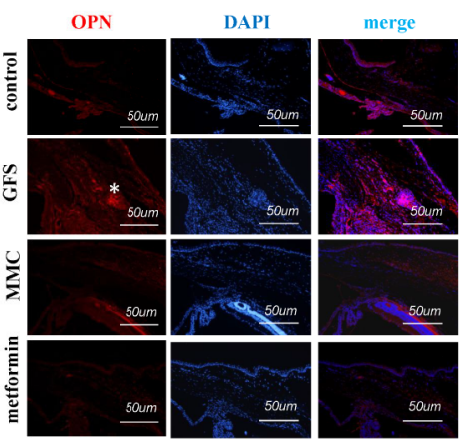

FIGURE 1 | Metformin prolongs filtering blebs survival in SD rats of GFS. (A) Schematic illustration of the experimental animal timeline. (B) Quantization of bleb area was analyzed. (C) H\&E and (D) Masson's trichrome were assessed for the fibrotic levels of filtrating blebs. (E-G) Immunohistochemical staining images showing the expression of collagen-1, vimentin, $\alpha$-SMA and OPN in filtrating bleb tissues at postoperative day 14. $n=10$. Data shown as mean values \pm SD. ${ }^{\star \star \star}$ denotes $p<0.001$. 
metformin treatment. $\alpha$-SMA, vimentin, collagen-1, OPN, fibronectin and CD31 were expressed higher levels in GFS group than metformin group with immunofluorescence staining (Figures 1E, F and Supplemental Figures S1D, E) (as white stars showed). Metformin significantly reduced the protein expression of OPN compared to GFS group, suggesting that the existence of lighter inflammatory response at surgery sites (Figure 1G) (as white stars showed). Therefore, the above results indicate that metformin reduces fibrosis in rats of GFS.

\section{AMPK Is Critical for Fibrosis in TGF- $\beta 2$ - Induced HConFs}

AMPK, a key sensor of cellular bioenergy, can control the conversion from anabolism to catabolism. Besides, AMPK is a known critical factor for modulating the pathological fibrosis process in terms of sensing and solving the resistance of mitochondrial ROS and maintaining cellular metabolic balance (Park et al., 2012; Rangarajan et al., 2018). Postoperative 2 weeks in SD rats GFS model, the immunohistochemical images showed that the level of $\alpha$-SMA at the wound site (GFS group) was significantly increased, but with a lower activated level of AMPK, as evidenced by decreased the phosphorylated level of Thr172 AMPK (Figure 2A) (as red/white stars showed). AICAR, an AMPK activator, prevented collagen-I, fibronectin, and $\alpha$-SMA expressions in TGF- $\beta 2(4 \mathrm{ng} / \mathrm{ml})$ treated HConFs cells (Figures 2B, C). Besides, flow cytometry results showed that AICAR effectively reduced TGF- $\beta 2$-induced the increase of ROS (Figure 2D). Meanwhile, AICAR increased the levels of antioxidants relative proteins/genes of $\gamma$-GCS, SOD1, SOD2 (Figures 2E, F) and significantly reduced the migration in HConFs after TGF- $\beta 2$ pretreatment (Figure 2G). The above results indicate that AICAR effectively inhibit TGF- $\beta 2$-induced cell proliferation and oxidative stress.

Metformin is also an AMPK activator; besides, it has been widely applied as a hypoglycemic agent in clinical, without any obvious complications (Supplemental Figure S2A). Here, we found that metformin could effectively reduce fibrotic and antioxidative relative proteins/genes level at a concentration of 2
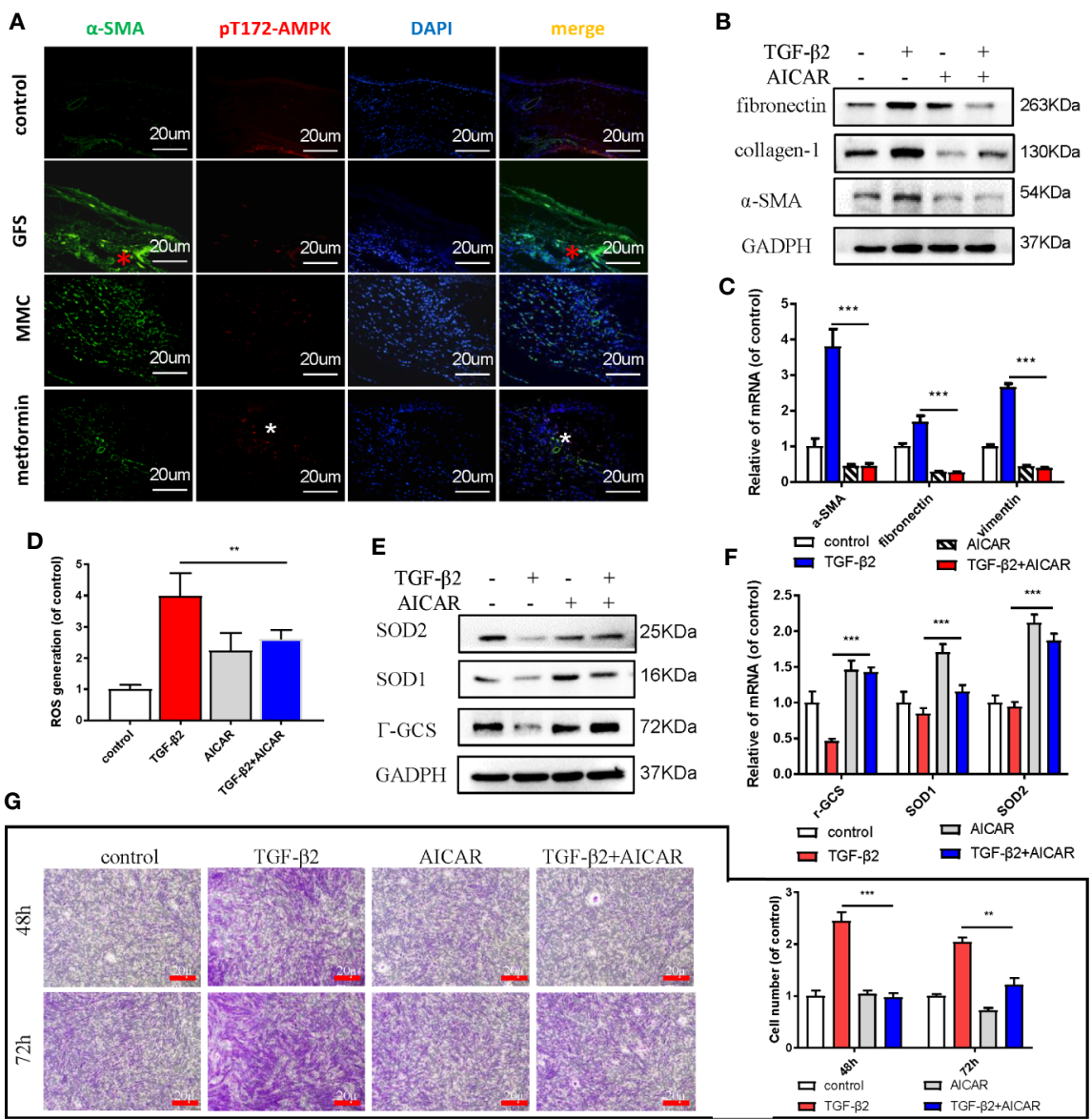

FIGURE 2 | AICAR inhibits TGF- $\beta 2$-induced HConFs fibrosis. (A) The expression of Thr172 AMPK phosphorylation in filtrating blebs tissues in rats were analyzed by historical images. (B, C) The fibrotic levels in TGF- $\beta 2$ treated HConFs followed by AICAR treatment were determined by western blotting/qRT-PCR. (D) The ROS levels were analyzed by flow cytometry assay. (E) Relative antioxidants proteins and (F) genes were determined. (G) The migration capabilities of HConFs were assessed by transwells. $n=3$. All data shown as mean values \pm SD. ** and ${ }^{\star \star \star}$ denote $p<0.01$ and $p<0.001$, respectively. 
$\mathrm{mM}$ in TGF- $\beta 2$ treated HConFs followed by metformin treatment at different concentrations (Figures 3A-D). Similarly, metformin was as effective as AICAR $(1 \mathrm{mM})$ in reducing increased ROS levels and decreased the migration of TGF- $\beta 2$-induced HConFs (Figures 3E, F). To further analyze the role of metformin in HConFs proliferation, we found that metformin significantly inhibited cells proliferation in the G0/G1 phase by flow cytometry assay (Figures $\mathbf{4 A}, \mathbf{B}$ ). The CCK-8 assay results showed that metformin $(2 \mathrm{mM})$ could effectively inhibit TGF$\beta 2$-induced cell proliferation (Figure 4C). Representative images showing immunofluorescence staining for fibronectin showed that AICAR or metformin effectively reduced related fibrotic levels (Supplemental Figures S3A, B). Furthermore, metformin reversed the expression of proliferative proteins and genes such as CyclinD1, CKD4, P21, P27 (Figures 4D, E), indicating that the activation of AMPK may promote antioxidant capacity and inhibits the cells migration in HConFs induced by TGF- $\beta 2$. Therefore, the above results suggest that metformin, likes
AICAR, may reduce TGF- $\beta 2$-induced HConFs' fibrosis, migration and proliferation by activating AMPK.

\section{Metformin Reverses Fibrosis in TGF- $\beta 2$ - Induced HConFs by Activating AMPK/Nrf2 Axis}

Previous literatures reported that the activation of AMPK/Nrf2 pathway ameliorated hepatic damage via decreasing the inflammation, oxidative stress and fibrosis levels in T2DM and PA-induced oxidative stress and cell injury (Wang et al., 2017; Kim et al., 2019). Activating Nrf2 may increase the level of multiple transcription factors related to anti-inflammatory, antioxidants and other cell protection pathways by combining with antioxidant response elements (Shanmugam et al., 2017; Li et al., 2020). Here, we hypothesized that metformin increased the intracellular ROS scavenging capacity and inhibited proliferation of TGF- 32 -induced HConFs by regulating the AMPK/Nrf2

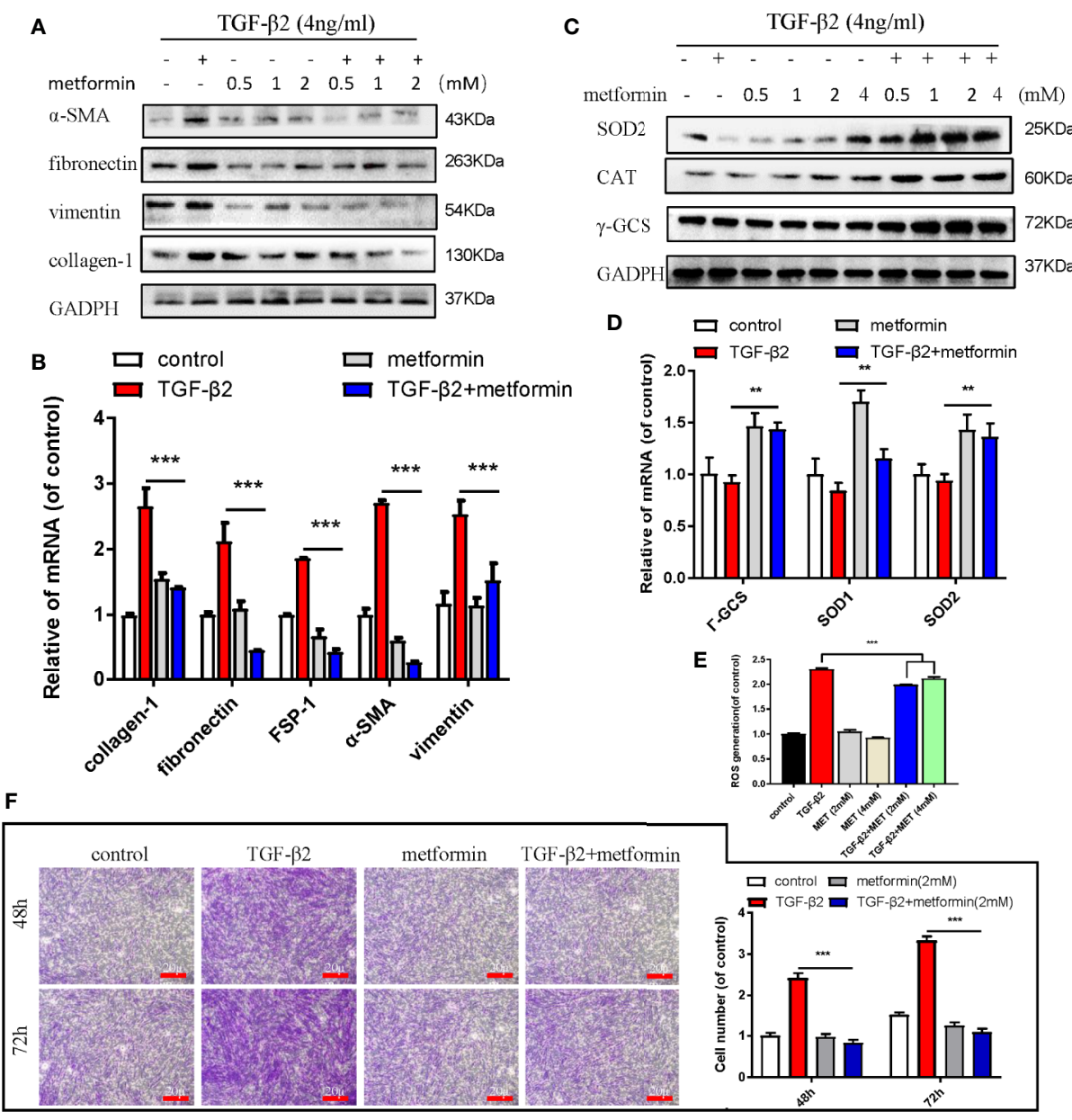

FIGURE 3 | Metformin reduces TGF- $\beta 2$-induced HConFs fibrosis. (A, B) The anti-fibrotic effects of metformin on TGF- $\beta 2$ treated HConFs were analyzed by western blot/qRT-PCR. (C, D) The antioxidative effect of metformin on TGF- $\beta 2$-induced HConFs was examined. (E) The ROS levels of HConFs were assessed by flow cytometry. (F) The migration capability of TGF- $\beta 2$-induced HConFs followed by metformin treatment were analyzed by transwells and statistical analysis. $\mathrm{n}=3$. All data shown as mean values $\pm \mathrm{SD}$. ${ }^{\star \star}$ and ${ }^{\star \star \star}$ denote $\mathrm{P}<0.01$ and $\mathrm{P}<0.001$, respectively. 
A
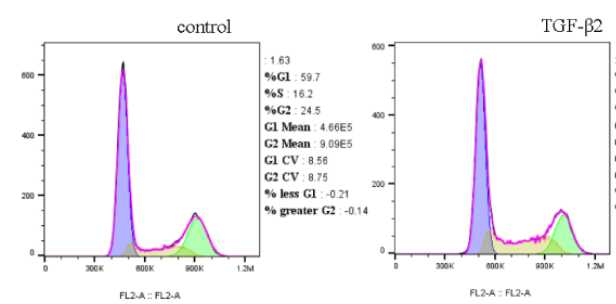

GF- $\beta 2$
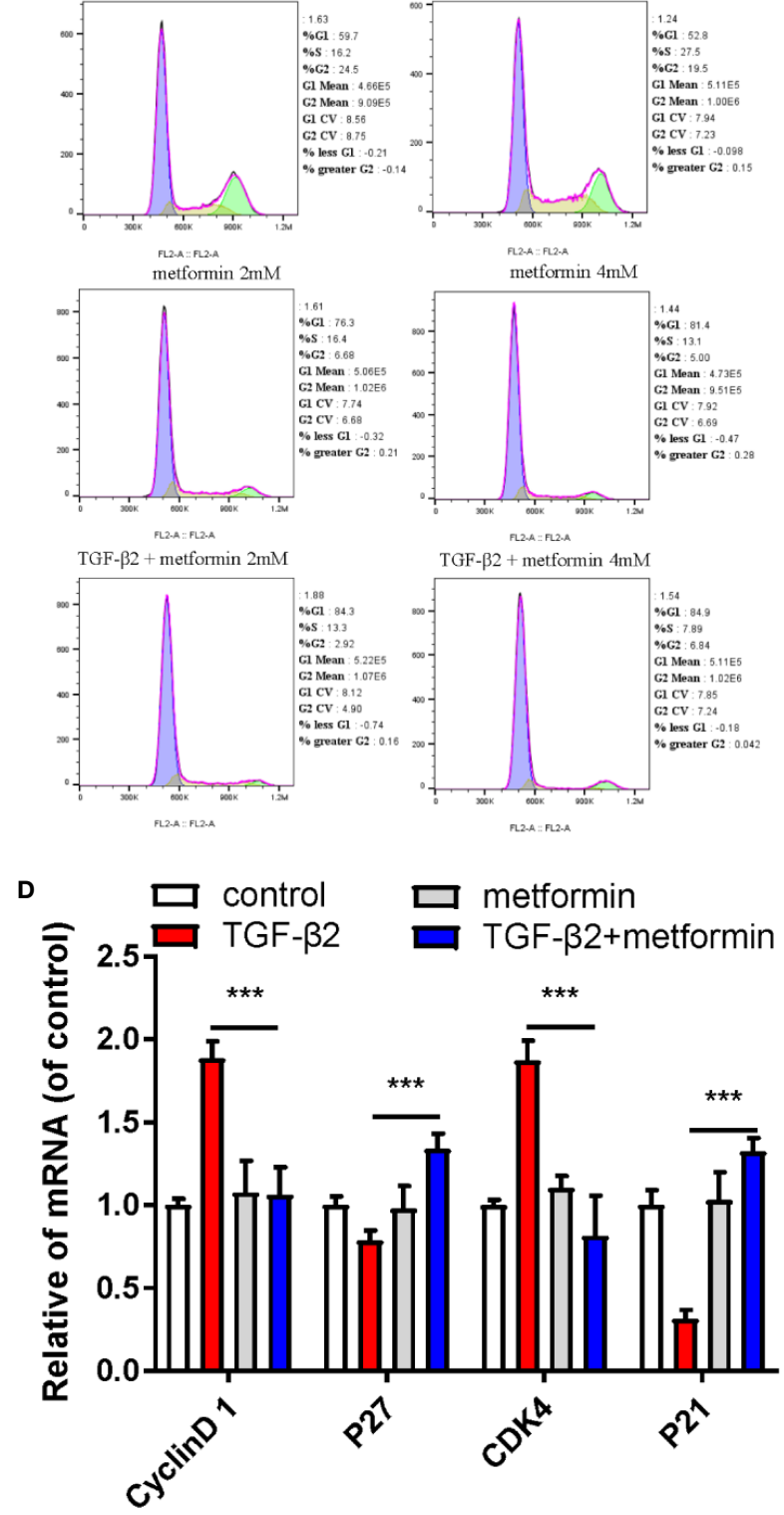

B
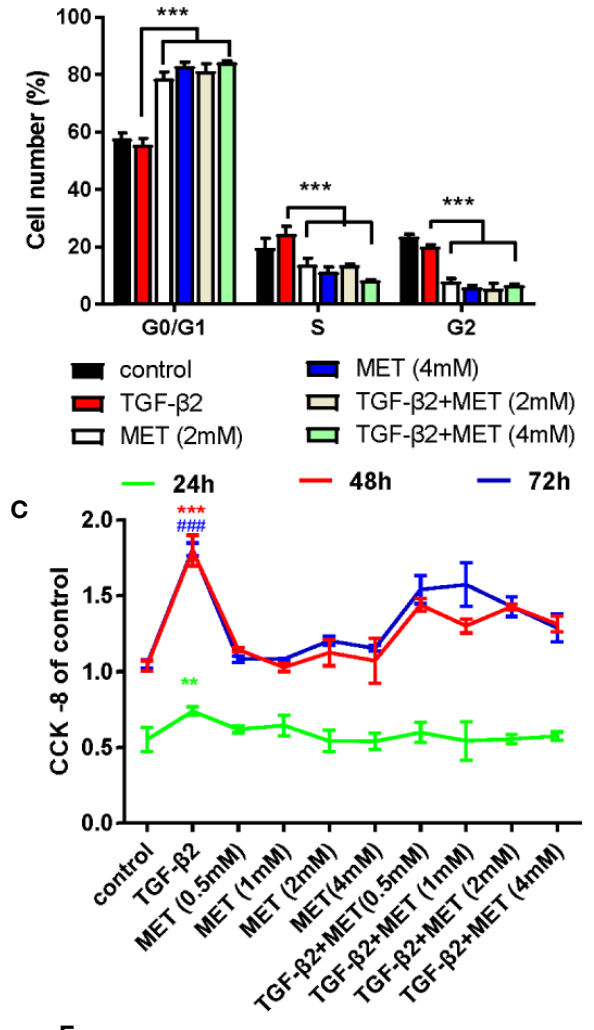

E

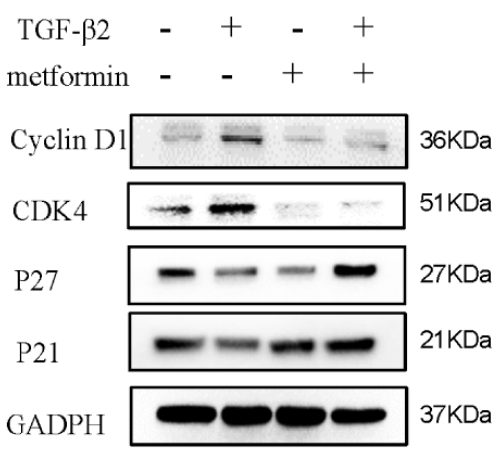

FIGURE 4 | Metformin induces cell cycle arrest at G0/G1 phase in HConFs. (A) Cell cycle was analyzed by flow cytometry assay and (B) quantization of the rates in HConFs cells. (C) CCK8 was used to analyze the proliferation of HConFs. (D) The genes levels of CDK4, P21, CyclinD1 and P27 were assessed by qRT-PCR.

(E) The relative cell cycle proteins level of CDK4, CyclinD1, P21 and P27 were tested by Western blotting. ${ }^{* \star} p<0.01,{ }^{\# \# \# / * \star} p<0.001$. MET $=$ Metformin.

signaling pathway. In vitro, we found that AICAR elevated the phosphorylation expressions of ACC and AMPK, but inhibited the level of p-smad2/3 (Figure 5A), and the effects of metformin at $2 \mathrm{mM}$ doses on HConFs induced by TGF- $\beta 2$ was consistent (Figure 5B). On the contrary, silencing AMPK mediated the constitutive expression of fibronectin in cells after TGF- $\beta 2$ treatment. Precondition with Compound C (an AMPK inhibitor) or ML 385 (a Nrf2 inhibitor) not only reversed metformin arrested cells in the G0/G1 phase (Figures $\mathbf{5 C}-\mathbf{F}$ ), but also decreased the capacity of metformin increased intracellular antioxidants degree on proteins and genes (Figures 5G-I). In Figures 5J-L, the relative fibrotic proteins and gens in TGF- $\beta 2$-treated HConFs with pretreated by metformin were significantly increased followed by Compound C or ML 385 treatment. Together, the above experiments indicate that metformin reduces the levels of TGF- $\beta 2$-induced HConFs oxidative stress, proliferation, fibrotic proteins/genes via activating AMPK/Nrf2 signaling pathway. 


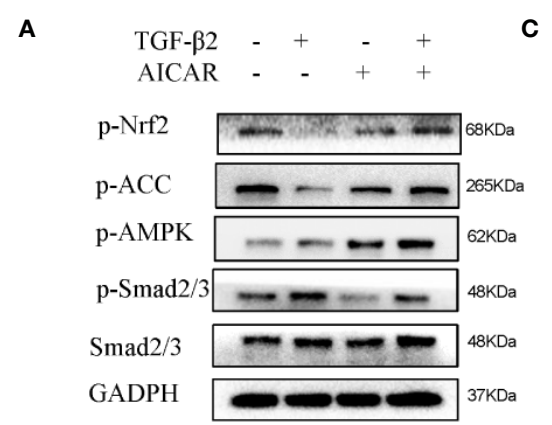

B $\quad$ TGF- $\beta 2(4 \mathrm{ng} / \mathrm{ml})$

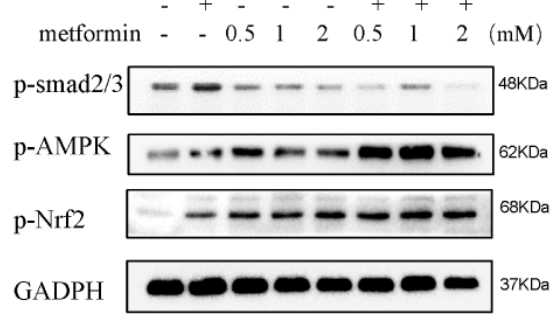

$\mathbf{F}$

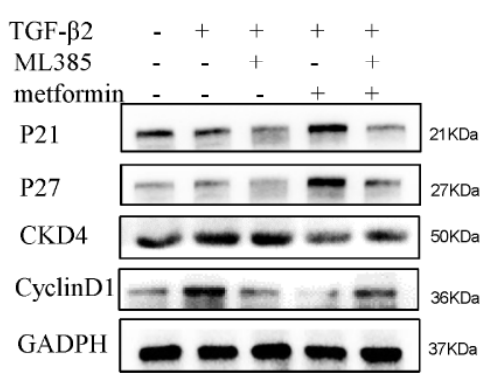

I

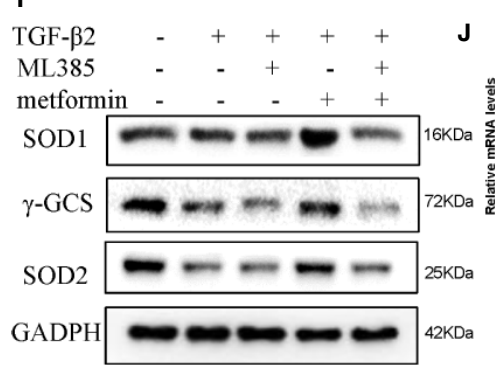

G

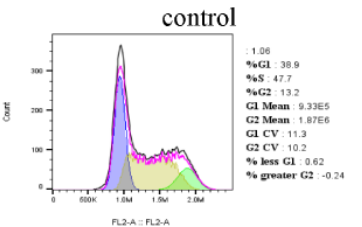

TGF- $\beta 2+\mathrm{MET}+$ Copound C
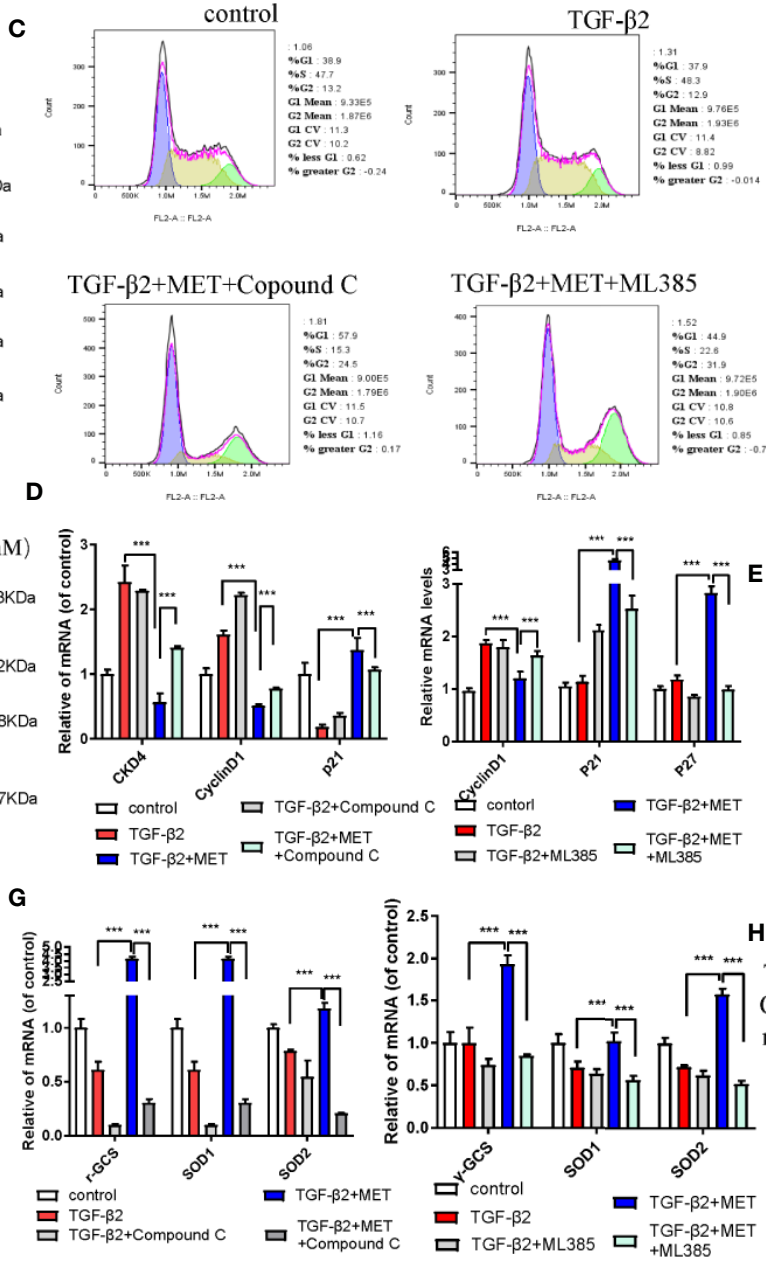

TGF- $\beta 2+\mathrm{MET}+\mathrm{ML} 385$
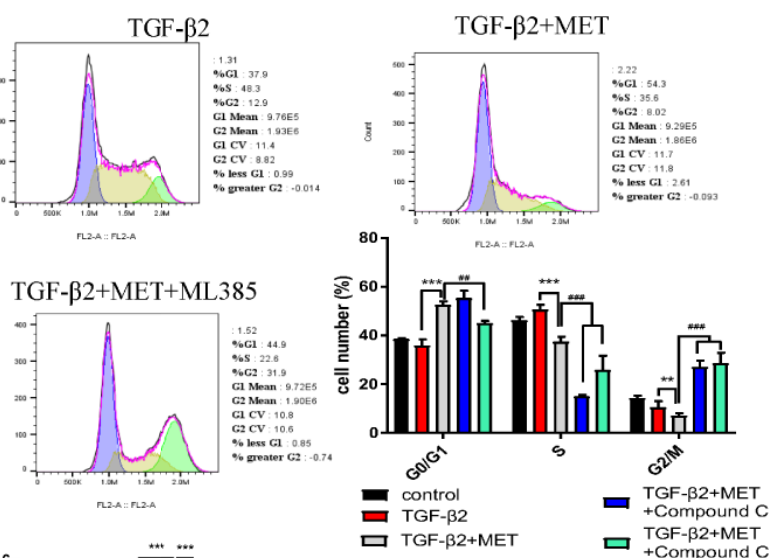

$T \mathrm{TF}-\beta 2+\mathrm{ME}$ 口 TGF- $32+M E T$

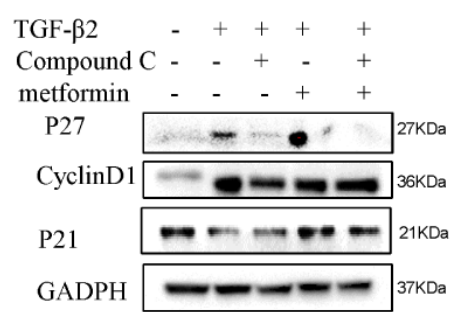

H

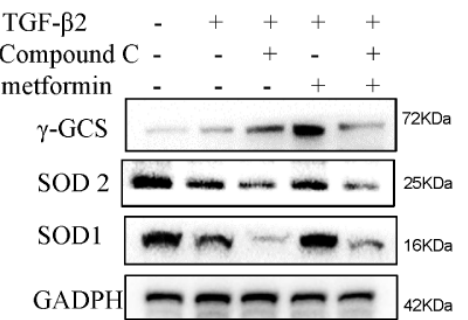

TGF- $\beta 2$

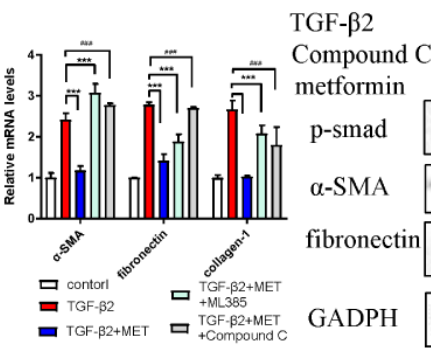

L

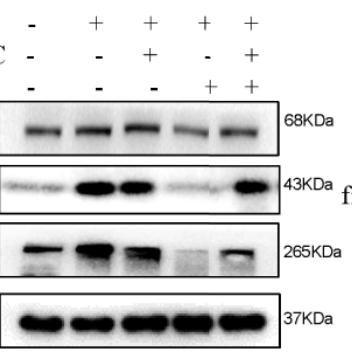

TGF- $\beta 2$

ML385

$\begin{array}{llllll}\mathrm{ML385} & - & - & + & - & + \\ \text { metformin } & - & - & - & + & +\end{array}$

fibronectin $=2-m$

$\alpha$ - SMA $=-m-m$

GADPH p-smad $=-m-\infty \mathrm{KDa}$

FIGURE 5 | Metformin reduces fibrosis in HConFs pretreated by TGF- $\beta 2$ via activating AMPK/Nrf2 axis. (A-B) AICAR or metformin could increase the levels of $p-$ Nrf2(S40), Nrf2 and p-AMPK in HConFs after TGF- $\beta 2$ precondition for 24 h. (C) Cell cycle was tested and quantization of the rates in HConFs cells. Compound C or ML385 could reverse the inhibition of metformin on HConFs' proliferation. (D-F) Cell cycle relative proteins/genes (CDK4, CyclinD1, P21 and P27) were detected by qRT-PCR/western blotting. (G-I) Antioxidant defense relative protein/genes $\gamma$-GCS, SOD1/2 and (J-L) fibrotic proteins/genes collagen-1, fibronectin, $\alpha$-SMA level in HConFs treated by metformin with/without Compound C $(10 \mu \mathrm{mol} / \mathrm{L})$ or ML385 $(10 \mathrm{mM})$ treatment for $24 \mathrm{~h}$. MET $=$ metformin, $\mathrm{n}=3$. All data shown as mean values \pm SD. ${ }^{\star \star / \# \# \#}$ and ${ }^{\star \star \star \star \# \# \# ~}$ denote $\mathrm{P}<0.01$ and $\mathrm{P}<0.001$, respectively.

\section{Organic Cation Transporter (OCT) Mediates the Uptake of Metformin in HConFs Cell}

Topical administration is the most convenient and patient compliant route of drug administration (Boddu et al., 2014). Metformin, a cation substrate of OCT, is one of few of the ophthalmic drugs that are transported by OCTs (atropine/quinidine), which reduce the uptake of cation substrates (Horie et al., 2011; Nirmal et al., 2012). For further study the effects of metformin on prolonging the survival of functional filtering blebs, the cation substrates of atropine/ quinidine were used in this study. As shown in Figures 6A, B, 


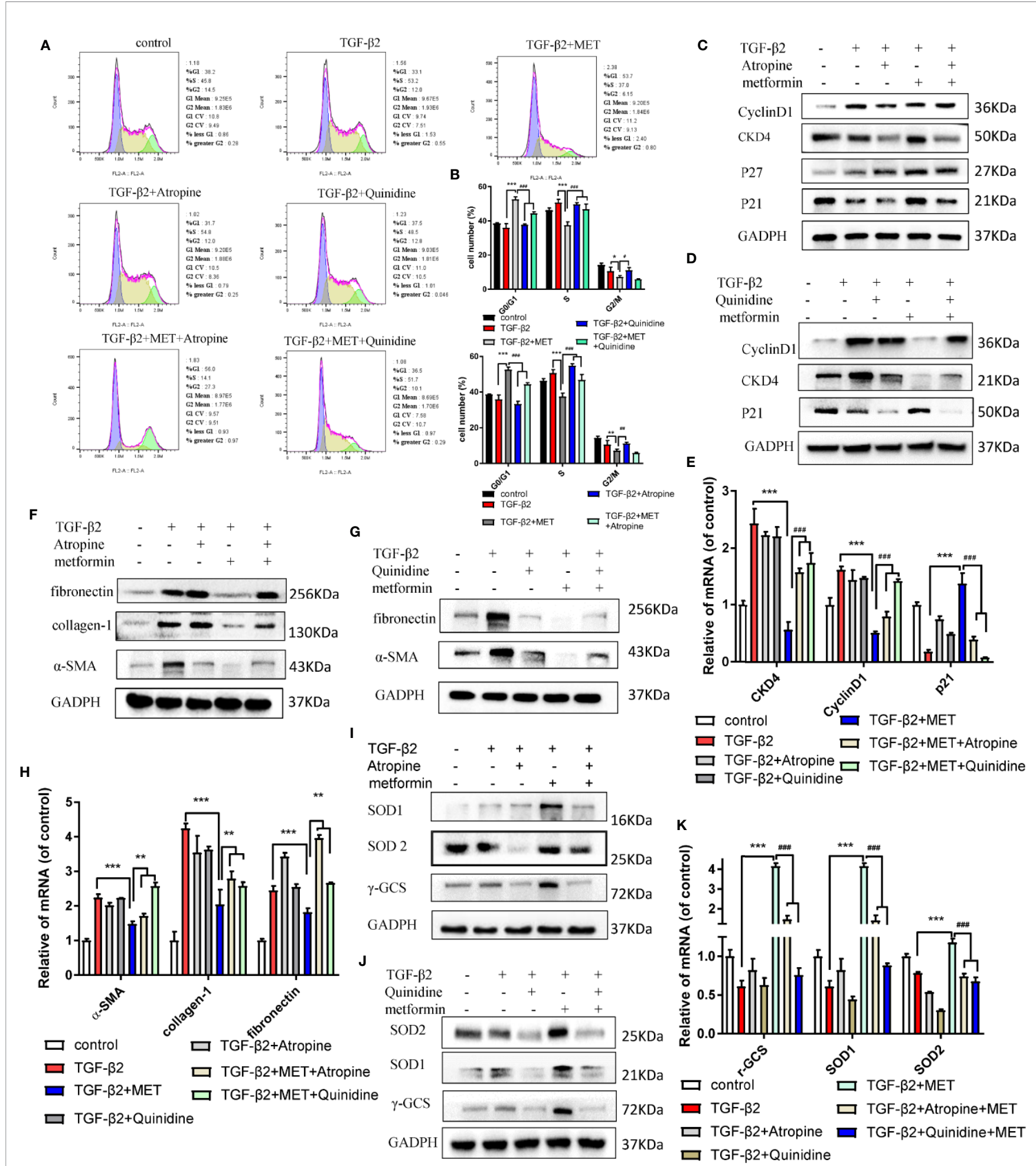

FIGURE 6 | The inhibitor of OCTs could reduce the capability of anti-proliferation of metformin. (A) Cell cycle was assessed by flow cytometry assay and (B) quantization of the rates in HConFs. (C-E) Cell cycle relative proteins/genes (CyclinD1, CDK4, P21 and P27) level were assessed in HConFs after Atropine/Quinidine treatment by qRT-PCR and western blot. (F-H) Relative fibrotic proteins/genes were assessed. (I-K) Antioxidants related proteins and genes ( $\alpha$-SMA, collagen-1, fibronectin, $\mathrm{N}$-cadherin and vimentin) were assessed in HConFs after Atropine/Quinidine treatment by qRT-PCR or western blotting. MET $=$ metformin, $n=3$. Data

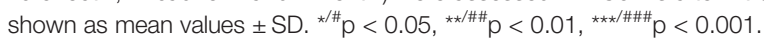


atropine/quinidine significantly reversed metformin arrested HConFs at G0/G1 phase. The relative proteins and genes level of Cyclin D1, CKD4, P27 and P21 were reversed in TGF- $\beta 2$-induced HConFs with metformin treatment followed by atropine/quinidine pretreatment (Figures 6C-E). At the same fibrotic and antioxidative proteins/genes level, atropine/quinidine significantly reduced the ability of metformin to resist fibrosis and improve antioxidant stress (Figures 6F-K). From the above results, we could conclude that the basis of metformin inhibit TGF- $\beta 2$-induced HConFs fibrosis is transferring into cells by OCTs.

\section{Metformin May Reverse the Pro-Fibrosis Effect of Different Macrophages Polarization on HConFs}

In the process of fibrotic diseases, macrophages exerted bidirectional effects on regulating matrix deposition and resolution, which were also central players in tissues' fibrosis (Ma et al., 2017; Conti et al., 2018; Han et al., 2018a). Macrophages could be polarized to the classical activation (M1) or alternative activation (M2) phenotype in the local immune microenvironment. Besides, ROS could damage the healing of skin wounds in the inflammatory stages through regulate the local microenvironments (Milich et al., 2019; Potter et al., 2019). Here, we found that topical application of metformin (7.85 mM/drops, four times/day) significantly inhibited the filtrating blebs scar formation in rats of GFS. There was a stronger immunofluorescence signaling of $\alpha$-SMA and M1 macrophage phenotype in GFS group compared to the other group (Figures 7A, B) (as white stars/arrows showed). To study the fibrotic effects of metformin on macrophages to HConFs, the co-culture of macrophages of different polarities (M0, M1 and M2) and HConFs at different times were analyzed, the results showed that macrophages of different polarities showed different induction responses to HConFs at $24 \mathrm{~h}$ (Supplemental Figure S4A), which were intricated and showed a lower level of fibrosis in general. Continue to observe co-culture for 48 and $72 \mathrm{~h}$, we found that metformin reduced the fibrotic genes/proteins expression of HConFs in most M1 macrophages (induced by LPS of $100 \mathrm{ng} / \mathrm{ml}$ ), and metformin could partially reduce the pro-fibrosis of HConFs cells by M2 macrophages (IL-4 + IL-13, $20 \mathrm{ng} / \mathrm{ml}$ ) genes maker expression (Figures 7C, E). Moreover, transwells assay showed metformin induced macrophage with IL-4 + IL-13 treatment increasing the cells migration in HConFs after co-culture $48 \mathrm{~h}$. While co-culture $72 \mathrm{~h}$, metformin reduced the cells migration and proliferation of HConFs (Figures 7D, G). To know that metformin interfered with the induction of HConFs fibrosis by macrophages of different polarities, metformin was added simultaneously during the induction of different polarities of macrophages, and RNA samples were collected for $12 \mathrm{~h}$. The data indicated that metformin reduced the phenotypic changes of M1 macrophages with pro-inflammatory effects, and decreased the genes level of some inflammatory factors. In addition, metformin also increased the expression of M2 macrophages with antiinflammatory effects (Figure 7F). Moreover, metformin obviously promoted the migration of M0, M1 and M2, indicating that metformin could promote the capability of macrophages (Supplementary Figure S4B). These antiinflammatory effects were supported by previous reports (Koh et al., 2018), suggesting that the anti-inflammatory capability of metformin reduced fibrosis in TGF- $\beta 2$-induced HConFs. Besides, the results may provide a novel direction that metformin approve local microenvironments to increase success GFS rates.

\section{DISCUSSIONS}

Glaucoma is the leading cause of irreversible blindness and reduces the vision-related quality of life worldwide (Crish and Calkins, 2015; Williams et al., 2017). GFS is recognized as the most effective option for reducing IOP. However, intraoperative tissue damage may stimulate the conjunctival fibroblasts migration, proliferation and differentiate from fibroblasts to myofibroblasts (Lanzl et al., 2016) that is an important process of wound repair, but usually leads to scarring (Wick et al., 2013). Current clinical anti-conjunctival scar drugs such as MMC or 5FU were widely applied to improve the outcomes of GFS, but are limited due to some serious complications (such as macular degeneration, filtered vesicle leakage and corneal epithelial dysfunction) (Cabourne et al., 2015). Thus, there is an urgent need for safer and more effective anti-scarring dugs in the clinic.

Accumulated studies have suggested that the effectively therapeutic role of metformin in non-diabetic diseases and has anti-fibrotic and anti-inflammatory effects (Faggi et al., 2018). Oral administration of metformin had protective effects on reducing in risk of developing OAG and had profound effects on anti-inflammatory and anti-angiogenesis on retinal vasculature (Lin et al., 2015; Han et al., 2018b). Besides, the transcorneal penetration of single topical administration metformin was significantly decreased with topical pretreatment of OCT blockers (Atropine or Quinidine) (Wang et al., 2008). These studies provided evidences that a potential therapeutic effect of eye drops of metformin in patients with GFS. In this study, we found that eye drops of metformin could effectively extend postoperative filtrating blebs survival and reduced the inflammatory response of conjunctival flap in rats' GFS model. Through immunohistochemical staining analysis of postoperative conjunctival tissues and surrounding vascular tissues, the expression of AMPK activation in damaged tissue was lower, while the protein of $\alpha$-SMA was overexpressed than normal group. According to previous reports, the damaged tissues were affected by inflammatory factors such as plateletderived growth factor PDGF, TGF- $\beta$ and hormones induction inhibited the expression of AMPK, resulting in damaged cell metabolism, abnormal proliferation, and eventually scar formation (Mishra et al., 2008; Foo et al., 2011; Rangarajan et al., 2018). Whether metformin can inactivate differentiated fibroblasts and resolve fibrosis in HConFs are still unclear. In this study, as expected that AICAR activating AMPK could effectively inhibit the proliferation, migration and phenotypic transformation of $\mathrm{HConFs}$ in a stressed state after injury. 
A
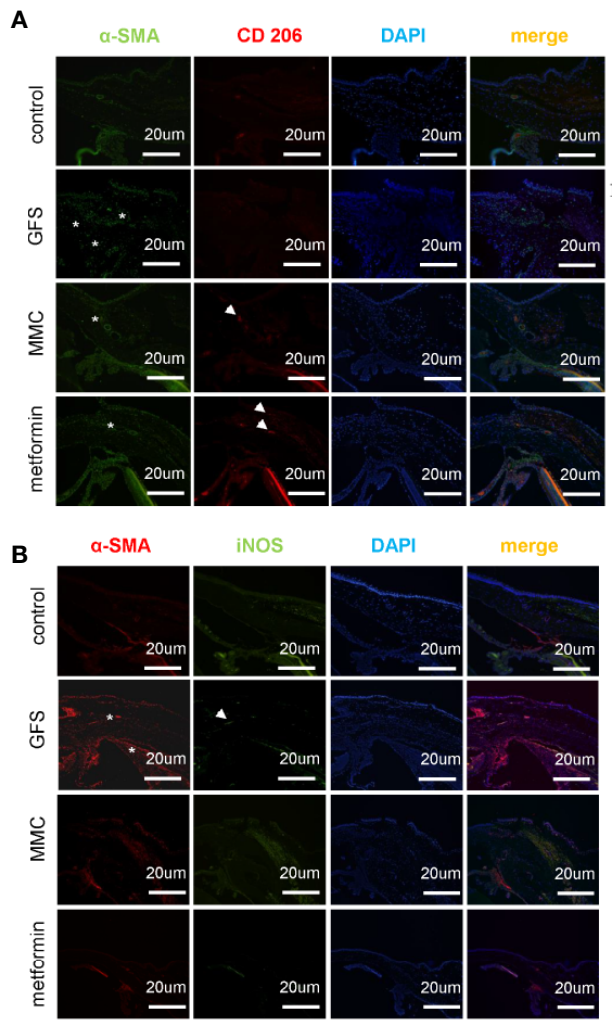

$\mathbf{F}$

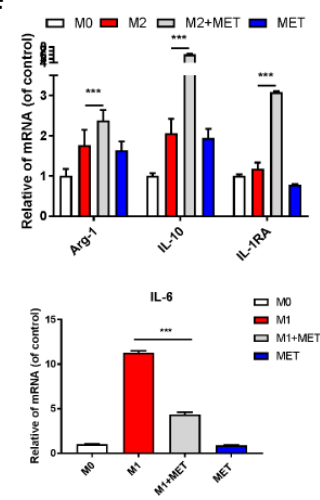

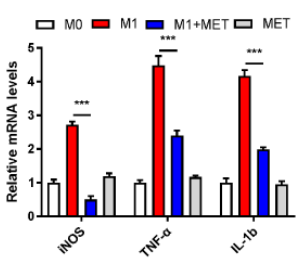

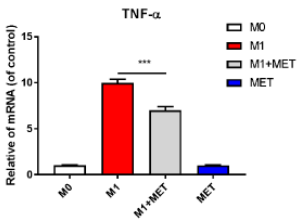

C
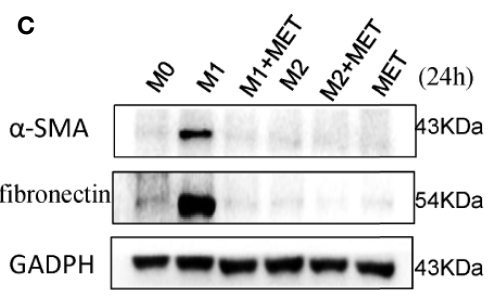

D

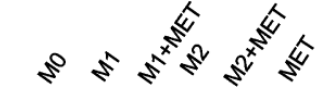

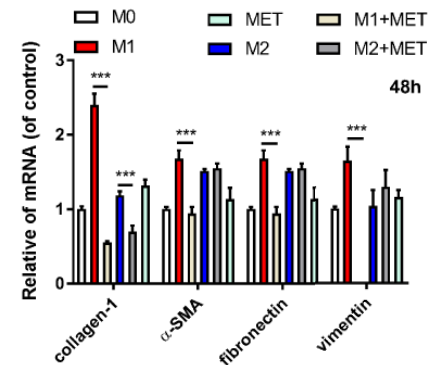

口 MO

口 MET 口 M1+MET

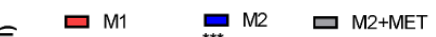
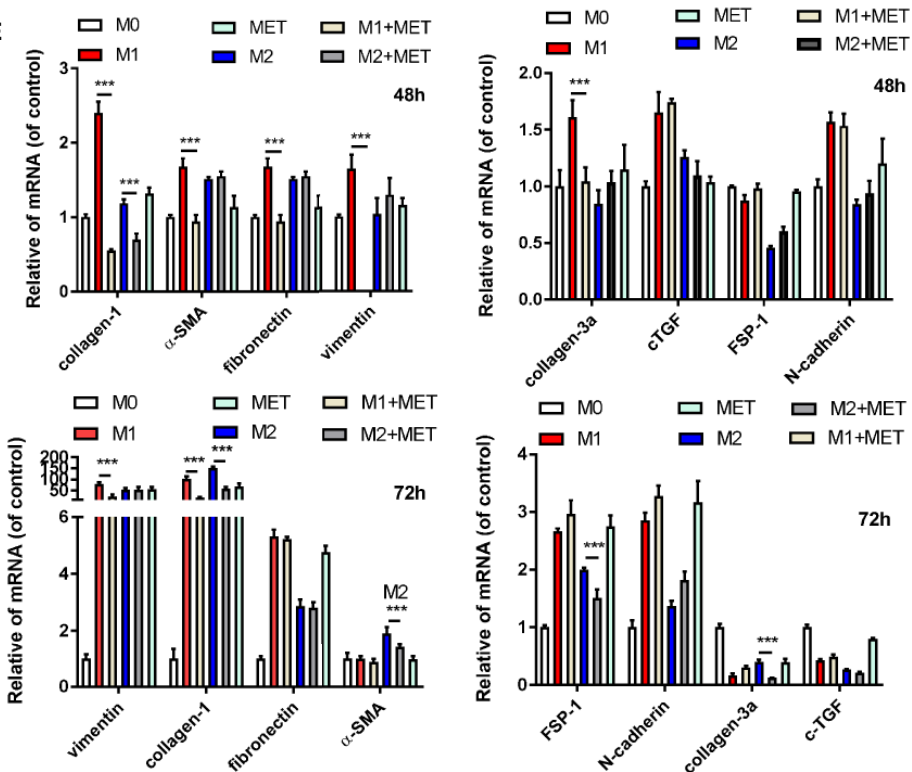

G

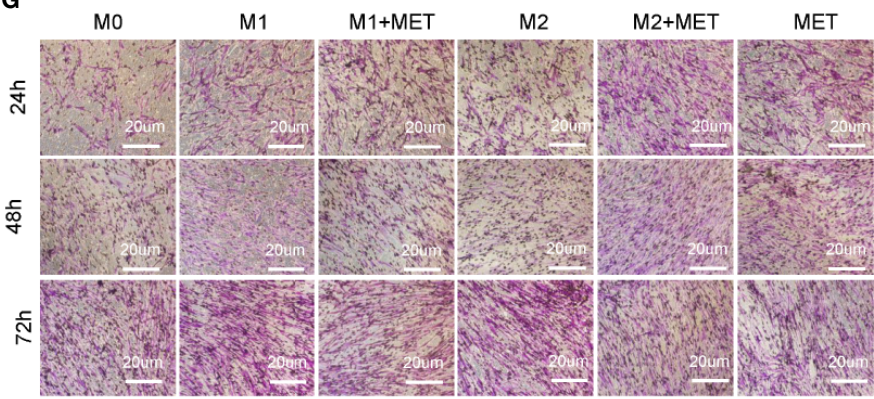

FIGURE 7 | Metformin reduces the fibrotic levels of HConFs via regulating macrophage polarization. (A, B) Immunofluorescence co-staining of $\alpha$-SMA and CD 206/ iNOS in filtrating blebs tissues. (C-E) The fibrosis or proliferation relative genes and proteins level of HConFs co-cultured with macrophage. (F) Quantification of iNOS, IL-6, TNF- $\alpha$ and IL-1 $\beta$ in M1-polarized macrophages (or M2-polarized macrophages) with/without metformin precondition by qRT-PCR. (G) The migration capability of HConFs co-cultured with MO, M1- and M2-polarized macrophages followed by metformin treatment were analyzed by transwells. MET = metformin. Data shown as mean values \pm SD. $n=3,{ }^{\star \star \star} p<0.001$.

Metformin, the other AMPK activator, also appeared to inhibit TGF- $\beta 2$-induced HConFs oxidation and proliferation, migration, and fibrosis. In addition to the important role of fibroblasts in scar formation, macrophages also played key role in tissue remodeling. In this experiment, metformin not only directly regulated the process of HConFs fibrosis, but also reversed the pro-fibrosis effect of different macrophages polarization on HConFs by reducing phenotypic expression of M1 macrophages with pro-inflammatory effects. For further study the mechanisms of metformin prolonged the survival of filtration blebs, we hypothesized that metformin could reduce TGF- $\beta 2$-induced $\mathrm{HConFs}$ by regulating the AMPK/Nrf2 signaling pathway to prolong the time of filtrating blebs. The proteins relevant to the AMPK/Nrf2 signaling pathway were assessed to test this hypothesis. Compound C (an AMPK inhibitor) and ML385 (a Nrf2 inhibitor) were used to verify the rationality of the pathway, As previous studies (Guo et al., 2018; Xie et al., 2020), the data indicated that Compound C/ML385 could effectively reverse the 


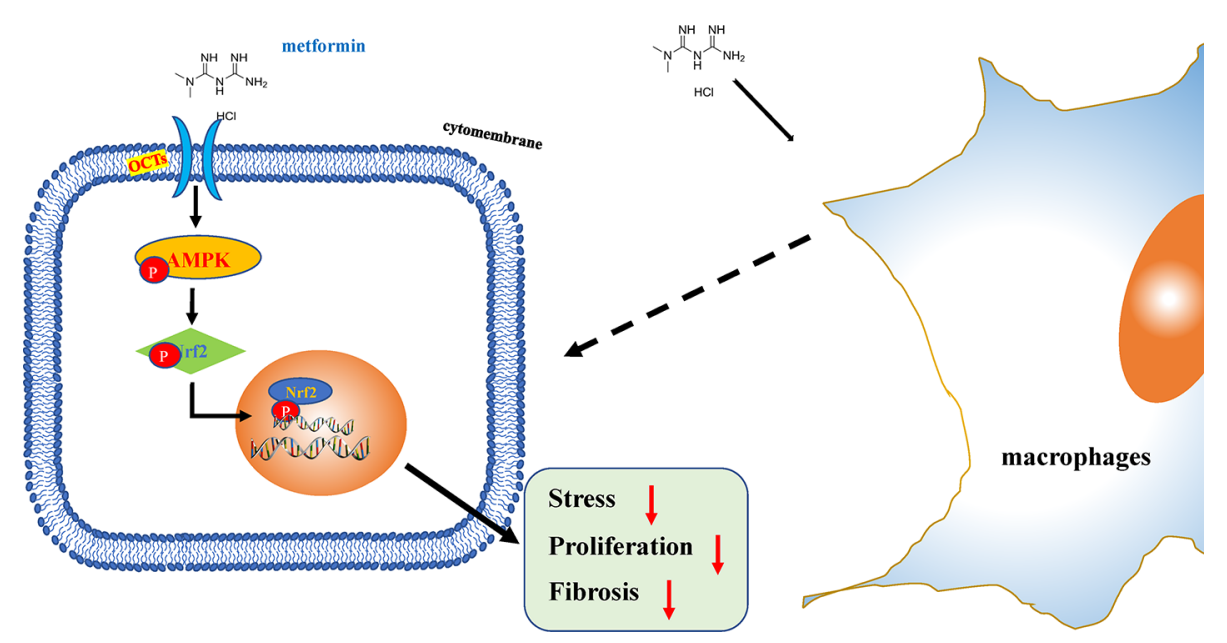

FIGURE 8 | Schematic illustration of the mechanism of metformin reduces oxidative stress and decreases proliferation of HConFs against fibrosis. Briefly, metformin obviously promotes antioxidant defense, inhibits proliferation via activating AMPK/Nrf2 axis and the downregulation of inflammatory biomarkers to improves issues repair potential.

inhibition of metformin on the proliferation, migration and fibrosis of TGF-induced HConFs.

Eye, an organ with a special anatomical location, is convenient for local application while effectively avoiding the blood-eye barrier (Nirmal et al., 2013b). The absorption of drugs by cells can effectively improve the efficacy of drugs under certain conditions. Moreover, organic cation transporters (OCT) inhibitor drugs can inhibit drug effects by competing with OCT, indicating that the drug-drug interaction by inhibition of OCT transporters may be important. Metformin, known as a cation substrate of OCT, transported by the hepatic and renal depends on OCTs (Nirmal et al., 2013a). Through the application of atropine/quinidine in this study, we found that OCTs were important for metformin therapeutic action in response to anti-fibrosis. Metformin could not only potentially reduce fibrosis by increasing the antioxidant capacity, but also reduced the proliferation of injured HConFs, and reversed the pro-fibrosis effect of different macrophages polarization on HConFs (Figure 8). However, due to the scarcity of clinical secondary surgery specimens, the analysis of AMPK expression in clinical patients has been limited.

In conclusion, our data indicate that metformin has a significant anti-fibrotic effect on GFS rats, indicating that metformin eye drops should be regarded as a therapeutic option for patients with GFS progressive fibrosis.

\section{DATA AVAILABILITY STATEMENT}

The datasets generated for this study are available on request to the corresponding authors.

\section{ETHICS STATEMENT}

The animal study was reviewed and approved by the National Institutes of Health and were approved by the Animal Care and Use Committee of Chongqing Medical University.

\section{AUTHOR CONTRIBUTIONS}

$\mathrm{XL}$ and YL performed the experiments. XL, QJ, ZW, LC, CZ, HW, PL, XY, YW, LX, YZ and CS interpreted data and drafted the manuscript. XL and YZ performed the data analysis. XL, LX, ZW and CShi conceived and designed the study.

\section{FUNDING}

This work was supported by the (Chongqing) National Natural Science Foundation of China (Grant nos. 81670860, 81470629 and No. cstc 2018jcyjAX0034), University Innovation Team Building Program of Chongqing (CXTDG201602020, to CShi), National Key Research and Development Program (2016YFC1000805, to CShi).

\section{SUPPLEMENTARY MATERIAL}

The Supplementary Material for this article can be found online at: https://www.frontiersin.org/articles/10.3389/fphar.2020. 01038/full\#supplementary-material 


\section{REFERENCES}

Addicks, E. M., Quigley, H. A., Green, W. R., and Robin, A. L. (1983). Histologic Characteristics of Filtering Blebs in Glaucomatous Eyes. Arch. Ophthalmol. 101, 795-798. doi: 10.1001/archopht.1983.01040010795021

Biondo, L. A., Batatinha, H. A., Souza, C. O., Teixeira, A. A. S., Silveira, L. S., Alonso-Vale, M. I., et al. (2018). Metformin Mitigates Fibrosis and Glucose Intolerance Induced by Doxorubicin in Subcutaneous Adipose Tissue. Front. Pharmacol. 9, 452. doi: 10.3389/fphar.2018.00452

Boddu, S. H., Gupta, H., and Patel, S. (2014). Drug delivery to the back of the eye following topical administration: an update on research and patenting activity. Recent Pat. Drug Delivery Formul. 8, 27-36. doi: 10.2174/1872211308666140130093301

Cabourne, E., Clarke, J. C. K., Schlottmann, P. G., and Evans, J. R. (2015). Mitomycin $\mathrm{C}$ versus 5-Fluorouracil for wound healing in glaucoma surgery. Cochrane Database Syt. Rev. 11, CD006259. doi: 10.1002/14651858.CD006259.pub2

Conti, P., Caraffa, A., Mastrangelo, F., Tettamanti, L., Ronconi, G., Frydas, I., et al. (2018). Critical role of inflammatory mast cell in fibrosis: Potential therapeutic effect of IL-37. Cell Prolif. 51, e12475. doi: 10.1111/cpr.12475

Crish, S. D., and Calkins, D. J. (2015). Central visual pathways in glaucoma: evidence for distal mechanisms of neuronal self-repair. J. Neuroophthalmol. 35 Suppl 1, S29-S37. doi: 10.1097/WNO.0000000000000291

Faggi, L., Giustina, A., and Tulipano, G. (2018). Effects of metformin on cell growth and AMPK activity in pituitary adenoma cell cultures, focusing on the interaction with adenylyl cyclase activating signals. Mol. Cell Endocrinol. 470, 60-74. doi: 10.1016/j.mce.2017.09.030

Foo, N. P., Lin, S. H., Lee, Y. H., Wu, M. J., and Wang, Y. J. (2011). alpha-Lipoic acid inhibits liver fibrosis through the attenuation of ROS-triggered signaling in hepatic stellate cells activated by PDGF and TGF-beta. Toxicology 282, 3946. doi: 10.1016/j.tox.2011.01.009

Guo, S., Meng, X. W., Yang, X. S., Liu, X. F., Ou-Yang, C. H., and Liu, C. (2018). Curcumin administration suppresses collagen synthesis in the hearts of rats with experimental diabetes. Acta Pharmacol. Sin. 39, 195-204. doi: 10.1038/aps.2017.92

Han, J., Kim, Y. S., Lim, M. Y., Kim, H. Y., Kong, S., Kang, M., et al. (2018a). Dual Roles of Graphene Oxide To Attenuate Inflammation and Elicit Timely Polarization of Macrophage Phenotypes for Cardiac Repair. ACS Nano 12, 1959-1977. doi: 10.1021/acsnano.7b09107

Han, J., Li, Y., Liu, X., Zhou, T., Sun, H., Edwards, P., et al. (2018b). Metformin suppresses retinal angiogenesis and inflammation in vitro and in vivo. PloS One 13, e0193031. doi: 10.1371/journal.pone.0193031

Hardie, D. G., Ross, F. A., and Hawley, S. A. (2012). AMPK: a nutrient and energy sensor that maintains energy homeostasis. Nat. Rev. Mol. Cell Biol. 13, 251262. doi: $10.1038 / \mathrm{nrm} 3311$

Horie, A., Sakata, J., Nishimura, M., Ishida, K., Taguchi, M., and Hashimoto, Y. (2011). Mechanisms for membrane transport of metformin in human intestinal epithelial Caco-2 cells. Biopharm. Drug Dispos. 32, 253-260. doi: 10.1002/bdd.755

Juban, G., Saclier, M., Yacoub-Youssef, H., Kernou, A., Arnold, L., Boisson, C., et al. (2018). AMPK Activation Regulates LTBP4-Dependent TGF-betal Secretion by Pro-inflammatory Macrophages and Controls Fibrosis in Duchenne Muscular Dystrophy. Cell Rep. 25, 2163-2176 e2166. doi: 10.1016/j.celrep.2018.10.077

Kim, Y., Lee, H., Kim, S. Y., and Lim, Y. (2019). Effects of Lespedeza Bicolor Extract on Regulation of AMPK Associated Hepatic Lipid Metabolism in Type 2 Diabetic Mice. Antioxid. (Basel) 8, 599-613. doi: 10.3390/antiox8120599

Koh, Y. C., Yang, G., Lai, C. S., Weerawatanakorn, M., and Pan, M. H. (2018). Chemopreventive Effects of Phytochemicals and Medicines on M1/M2 Polarized Macrophage Role in Inflammation-Related Diseases. Int. J. Mol. Sci. 19, 2208-2238. doi: 10.3390/ijms19082208

Lanzl, I. M., Poimenidou, M., and Spaeth, G. L. (2016). [Possibilities and limitations of eye drops for glaucoma therapy]. Ophthalmologe 113, 824832. doi: $10.1007 / \mathrm{s} 00347-016-0332-7$

Li, X., Leng, Y., Li, X., Wang, Y., Luo, P., Zhang, C., et al. (2020). The TßR IItargeted aptamer S58 prevents fibrosis after glaucoma filtration surgery. Aging 12, 8837-8857. doi: 10.18632/aging.102997

Lin, H. C., Stein, J. D., Nan, B., Childers, D., Newman-Casey, P. A., Thompson, D. A., et al. (2015). Association of Geroprotective Effects of Metformin and Risk of Open-Angle Glaucoma in Persons With Diabetes Mellitus. JAMA Ophthalmol. 133, 915-923. doi: 10.1001/jamaophthalmol.2015.1440

Ma, P. F., Gao, C. C., Yi, J., Zhao, J. L., Liang, S. Q., Zhao, Y., et al. (2017). Cytotherapy with M1-polarized macrophages ameliorates liver fibrosis by modulating immune microenvironment in mice. J. Hepatol. 67, 770-779. doi: 10.1016/j.jhep.2017.05.022

Milich, L. M., Ryan, C. B., and Lee, J. K. (2019). The origin, fate, and contribution of macrophages to spinal cord injury pathology. Acta Neuropathol. 137, 785797. doi: 10.1007/s00401-019-01992-3

Mishra, R., Cool, B. L., Laderoute, K. R., Foretz, M., Viollet, B., and Simonson, M. S. (2008). AMP-activated protein kinase inhibits transforming growth factor-betainduced Smad3-dependent transcription and myofibroblast transdifferentiation. J. Biol. Chem. 283, 10461-10469. doi: 10.1074/jbc.M800902200

Moro, M., Caiola, E., Ganzinelli, M., Zulato, E., Rulli, E., Marabese, M., et al. (2018). Metformin Enhances Cisplatin-Induced Apoptosis and Prevents Resistance to Cisplatin in Co-mutated KRAS/LKB1 NSCLC. J. Thorac. Oncol. 13, 1692-1704. doi: 10.1016/j.jtho.2018.07.102

Nirmal, J., Velpandian, T., Singh, S. B., Biswas, N. R., Azad, R., Thavaraj, V., et al. (2012). Evaluation of the functional importance of organic cation transporters on the ocular disposition of its intravitreally injected substrate in rabbits. Curr. Eye Res. 37, 1127-1135. doi: 10.3109/02713683.2012.715715

Nirmal, J., Singh, S. B., Biswas, N. R., Thavaraj, V., Azad, R. V., and Velpandian, T. (2013a). Potential pharmacokinetic role of organic cation transporters in modulating the transcorneal penetration of its substrates administered topically. Eye (Lond) 27, 1196-1203. doi: 10.1038/eye.2013.146

Nirmal, J., Sirohiwal, A., Singh, S. B., Biswas, N. R., Thavaraj, V., Azad, R. V., et al. (2013b). Role of organic cation transporters in the ocular disposition of its intravenously injected substrate in rabbits: implications for ocular drug therapy. Exp. Eye Res. 116, 27-35. doi: 10.1016/j.exer.2013.07.004

Park, C. S., Bang, B. R., Kwon, H. S., Moon, K. A., Kim, T. B., Lee, K. Y., et al. (2012). Metformin reduces airway inflammation and remodeling via activation of AMP-activated protein kinase. Biochem. Pharmacol. 84, 1660-1670. doi: 10.1016/j.bcp.2012.09.025

Patterson, R. E., Marinac, C. R., Sears, D. D., Kerr, J., Hartman, S. J., CadmusBertram, L., et al. (2018). The Effects of Metformin and Weight Loss on Biomarkers Associated With Breast Cancer Outcomes. J. Natl. Cancer Inst. 110, 1239-1247. doi: 10.1093/jnci/djy040

Potter, D. A., Veitch, D., and Johnston, G. A. (2019). Scarring and wound healing. Br. J. Hosp. Med. (Lond) 80, C166-C171. doi: 10.12968/hmed.2019.80.11.C166 Quarta, A., Le Blon, D., D'aes, T., Pieters, Z., Taj, S. H., Miró-Mur, F., et al. (2019). Murine iPSC-derived microglia and macrophage cell culture models recapitulate distinct phenotypical and functional properties of classical and alternative neuro-immune polarisation. Brain Behav. Immun. 82, 406-421. doi: 10.1016/j.bbi.2019.09.009

Rangarajan, S., Bone, N. B., Zmijewska, A. A., Jiang, S., Park, D. W., Bernard, K., et al. (2018). Metformin reverses established lung fibrosis in a bleomycin model. Nat. Med. 24, 1121-1127. doi: 10.1038/s41591-018-0087-6

Salminen, A., and Kaarniranta, K. (2012). AMP-activated protein kinase (AMPK) controls the aging process via an integrated signaling network. Ageing Res. Rev. 11, 230-241. doi: 10.1016/j.arr.2011.12.005

Sato, N., Takasaka, N., Yoshida, M., Tsubouchi, K., Minagawa, S., Araya, J., et al. (2016). Metformin attenuates lung fibrosis development via NOX4 suppression. Respir. Res. 17, 107. doi: 10.1186/s12931-016-0420-x

Schlunck, G., Meyer-ter-Vehn, T., Klink, T., and Grehn, F. (2016). Conjunctival fibrosis following filtering glaucoma surgery. Exp. Eye Res. 142, 76-82. doi: 10.1016/j.exer.2015.03.021

Shanmugam, G., Narasimhan, M., Tamowski, S., Darley-Usmar, V., and Rajasekaran, N. S. (2017). Constitutive activation of Nrf2 induces a stable reductive state in the mouse myocardium. Redox Biol. 12, 937-945. doi: 10.1016/j.redox.2017.04.038

Sharma, H., and Kumar, S. (2017). Natural AMPK Activators: An Alternative Approach for the Treatment and Management of Metabolic Syndrome. Curr. Med. Chem. 24, 1007-1047. doi: 10.2174/0929867323666160406120814

Sharma, P., and Kumar, S. (2018). Metformin inhibits human breast cancer cell growth by promoting apoptosis via a ROS-independent pathway involving mitochondrial dysfunction: pivotal role of superoxide dismutase (SOD). Cell Oncol. (Dordr) 41, 637-650. doi: 10.1007/s13402-018-0398-0

Sherwood, M. B., Esson, D. W., Neelakantan, A., and Samuelson, D. A. (2004). A new model of glaucoma filtering surgery in the rat. J. Glaucoma 13, 407-412. doi: 10.1097/01.ijg.0000131482.86547.5a

Sivalingam, V. N., Myers, J., Nicholas, S., Balen, A. H., and Crosbie, E. J. (2014). Metformin in reproductive health, pregnancy and gynaecological cancer: 
established and emerging indications. Hum. Reprod. Update 20, 853-868. doi: 10.1093/humupd/dmu037

Wang, Z. J., Yin, O. Q., Tomlinson, B., and Chow, M. S. (2008). OCT2 polymorphisms and in-vivo renal functional consequence: studies with metformin and cimetidine. Pharmacogenet. Genomics 18, 637-645. doi: 10.1097/FPC.0b013e328302cd41

Wang, L., Han, J., Shan, P., You, S., Chen, X., Jin, Y., et al. (2017). MD2 Blockage Protects Obesity-Induced Vascular Remodeling via Activating AMPK/Nrf2. Obes. (Silver Spring) 25, 1532-1539. doi: 10.1002/oby.21916

Wick, G., Grundtman, C., Mayerl, C., Wimpissinger, T. F., Feichtinger, J., Zelger, B., et al. (2013). The immunology of fibrosis. Annu. Rev. Immunol. 31, 107-135. doi: 10.1146/annurev-immunol-032712-095937

Williams, P. A., Marsh-Armstrong, N., and Howell, G. R. (2017). Neuroinflammation in glaucoma: A new opportunity. Exp. Eye Res. 157, 20-27. doi: 10.1016/j.exer.2017. 02.014

Xie, S., Deng, W., Chen, J., Wu, Q. Q., Li, H., Wang, J., et al. (2020). Andrographolide Protects Against Adverse Cardiac Remodeling After
Myocardial Infarction through Enhancing Nrf2 Signaling Pathway. Int. J. Biol. Sci. 16, 12-26. doi: 10.7150/ijbs.37269

Zada, M., Pattamatta, U., and White, A. (2018). Modulation of Fibroblasts in Conjunctival Wound Healing. Ophthalmology 125, 179-192. doi: 10.1016/ j.ophtha.2017.08.028

Conflict of Interest: The authors declare that the research was conducted in the absence of any commercial or financial relationships that could be construed as a potential conflict of interest.

Copyright (c) 2020 Li, Leng, Jiang, Wang, Luo, Zhang, Chen, Wang, Wang, Yue, Shen, Zhou, Shi and Xie. This is an open-access article distributed under the terms of the Creative Commons Attribution License (CC BY). The use, distribution or reproduction in other forums is permitted, provided the original author(s) and the copyright owner(s) are credited and that the original publication in this journal is cited, in accordance with accepted academic practice. No use, distribution or reproduction is permitted which does not comply with these terms. 